Pacific Journal of Mathematic 


\section{PRODUCTS OF FINITELY ADDITIVE SET FUNCTIONS FROM ORLICZ SPACES}

\section{VERNON ZANDER}

This note establishes two results on products of finitely additive vector-valued set functions from Orlicz spaces. A triple $(\Omega, \Sigma, \mu)$ is called a charge space if $\Sigma$ is a ring of subsets of a set $\Omega$ and the charge $\mu$ is a finitely additive, nonnegative, finite-valued function with domain $\Sigma$.

Theorem. For $\left(\Omega_{i}, \Sigma_{i}, \mu_{i}\right)(i=1, \cdots, n)$ a family of charge spaces and $(\Omega, \nu, \mu)$ the corresponding product charge space, for $u$ an $n$-linear continuous operator from the product of the Banach spaces $Z_{1}, \cdots, Z_{n}$ into a Banach space $W$, the function $v$ defined by $v(A)=u\left(v_{1}(A), \cdots, v_{n}(A)\right)$ for $A \in \Sigma$ and $v_{i}$ from the Orlicz space $A^{\Phi}\left(\Omega_{i}, \Sigma_{i}, \mu_{i}, Z_{i}\right)$ belongs to the Orlicz space $A^{\Phi}(\Omega, \Sigma, \mu, W)$.

For the infinite product case the following result holds:

Theorem. For $\left(\Omega_{t}, \Sigma_{t}, \mu_{t}\right)(t \in T)$ a family of probability charge spaces and $(\Omega, \Sigma, \mu)$ the product probability charge space, for $u$ an infinitely linear bounded operator on the multiplicative product space $P_{T}\left(A^{\Phi}\left(\Omega_{t}, \Sigma_{t}, \mu_{t}, Z_{t}\right), v_{t}^{\prime}\right)$ the function $v^{0}$ defined by $v^{0}(A)=u(v(A))$ for $A \in \Sigma$ belongs to the Orlicz space $A^{\Phi}(\Omega, \Sigma, \mu, W)$.

These results allow one to develop an integral determined by a product of charges from Orlicz spaces.

In a recent paper by Uhl [5] the Orlicz space $A^{\Phi}$ of vector-valued finitely additive set functions is investigated. The present paper presents results concerning finite and infinite products of finitely additive vector-valued set functions from the Orlicz spaces

$$
A^{\Phi}\left(\Omega_{r}, \Sigma_{r}, \mu_{r}, X_{r}\right)
$$

for $r$ ranging through an index set. The results for a finite product of set functions resemble a generalization of a result by Bogdanowicz [2,Th. 1] for the $L_{p}$-spaces of Lebesgue-Bochner summable functions; and the results for an infinite product of set functions resemble a generalization of a result for the Lebesgue space $L_{1}$ by Bogdanowicz and Zander [3, Proposition 5], but the techniques used for the results in the present paper are different.

We shall assume throughout that $\Phi$ is a convex, nondecreasing function defined on the positive real line such that $\Phi(0)=0$ and $\Phi$ is continuous except for at most one point, after which the function must be identically infinite. We shall also assume throughout that 
the function $\Phi$ satisfies the following growth condition:

$$
\Phi(x y) \leqq M \Phi(x) \Phi(y)
$$

for all $x, y \geqq 0$, where $M$ is a positive constant. (This growth condition is called the $\Delta^{\prime}$-condition (see $[4$, p. 29]).)

REMARK 1. Each of the functions $f(x)=x^{q}$ with $q \geqq 1$ and

$$
g(x)=x^{\alpha}\left(\log ^{+} x+1\right)
$$

with $\alpha>1$ satisfies the $\Delta^{\prime}$-condition with constant 1 , and each is a candidate for the function $\Phi$.

Let $\Omega$ be any set, $\Sigma$ a ring of subsets of $\Omega$, and $\mu$ a nonnegative, real-valued, finitely additive function with domain $\Sigma$. The associated triple $(\Omega, \Sigma, \mu)$ shall be called a charge space. If, further, $\Omega \in \Sigma$ and $u(\Omega)=1$ then the triple $(\Omega, \Sigma, \mu)$ shall be called a probability charge space.

For $(\Omega, \Sigma, \mu)$ a charge space and $Z$ a Banach space denote by $A^{\Phi}(\mu, Z)$ the space of all finitely additive functions $v$ from the ring $\Sigma$ into the space $Z$ which satisfy the following conditions: (i) $v$ vanishes on the $\mu$-null sets, (ii) $I \Phi(v / k) \leqq 1$ for some positive $k$, where the function $I_{\Phi}$ is defined by the following expression:

$$
I_{\Phi}(v)=\sup \Sigma\{\Phi(|v(A)| / \mu(A)) \mu(A): A \in \mathscr{F}\}
$$

where the supremum is taken over all finite families $\mathscr{F}$ of disjoint sets from the ring $\Sigma$. This definition is recently given by Uhl [5, p. 24], and is due originally to Bochner [1, p. 778]. By Theorem 11 of [5] the functional $N_{\Phi}$ defined by

$$
N_{\Phi}(v)=\inf \left\{k>0: I_{\Phi}(v / k) \leqq 1\right\}
$$

is a norm under which the space $A^{\Phi}(\mu, Z)$ is Banach space.

We shall use the following notation: Let $T_{i}$ be a family of subsets from an abstract set $X_{i}$, for $i=1, \cdots, n$. By $T_{1} \times \cdots \times T_{n}$ we shall mean the family of all sets of the form $A_{1} \times \cdots \times A_{n}$ where $A_{i} \in T_{i}$ for $i=1, \cdots, n$.

For charge spaces $\left(\Omega_{i}, \Sigma_{i}, \mu_{i}\right)(i=1, \cdots, n)$ define the triple $(\Omega$, $\left.\Sigma_{0}, \mu\right)$ by $\Omega=\Omega_{1} \times \cdots \times \Omega_{n}, \Sigma=\Sigma_{1} \times \cdots \times \Sigma_{n}, \Sigma_{0}$ is the ring generated from the prering $\Sigma$, and $\mu(A)=\mu_{1}\left(A_{1}\right) \cdots \mu_{n}\left(A_{n}\right)$ for $A \in \Sigma$. It is easy to see that $\left(\Omega, \Sigma_{0}, \mu\right)$ is a charge space.

Definition. For $\mathscr{F}$ a finite family of disjoint sets from the prering $\Sigma$ and for $i$ between 1 and $n$ let $\mathscr{F}_{i}$ denote the family of all $i$-th coordinate sets $A_{i}$, where $A_{1} \times \cdots \times A_{i} \times \cdots \times A_{n} \in \mathscr{F}$, and let 
$\mathscr{F}_{i}^{r}$ denote the refinement of $\mathscr{F}_{i}$ consisting of the finite family of disjoint sets from $\Sigma_{i}$ whose union is the same as that of all sets from $\mathscr{F}_{i}$. That is, if $\mathscr{F}_{i}=\left\{B_{1}, \cdots, B_{m}\right\}$, and if $B_{j}^{\prime}=\Omega_{i} \backslash B_{j}$ then $\mathscr{F}_{i}^{r}$ is the set of all intersections $C_{1} \cap \cdots \cap C_{m}$ where each $C_{j}$ is $B_{j}$ or $B_{j}^{\prime}$ and at least one $C_{j}$ is $B_{j}$. By the $\mathscr{F}$-product we shall mean the corresponding finite family of disjoint sets $\mathscr{F}_{1}^{r} \times \cdots \times \mathscr{F}_{n}^{r}$ from the prering $\Sigma$. We shall denote this family by $(\mathscr{F})$.

Lemma 1. For $v \in A^{\Phi}(\mu, Z)$ we have the relation

$$
I_{\Phi}(v)=\sup \Sigma\{\Phi(|v(A)| / \mu(A)) \mu(A): A \in(\mathscr{F})\}
$$

where the supremum is taken over all finite families $\mathscr{F}$ of disjoint sets from $\Sigma$.

Proof. Let $a$ denote the right side of the above relation. Then $I_{\mathscr{\phi}}(v) \geqq a$ since an $\mathscr{F}$-product is a particular finite disjoint family from $\Sigma$. Let $B, C$ be disjoint sets from $\Sigma$, and put $A=B \cup C$. Then the relation $I_{\mathscr{\phi}}(v) \leqq a$ follows from the positivity of values of the function $\Phi$ and from the relation

$$
\Phi(|v(A)| / \mu(A)) \mu(A) \leqq \Phi(|v(\beta)| / \mu(B)) \mu(B)+\Phi(|v(C)| / \mu(C)) \mu(C)
$$

which in turn follows from the convexity of the function $\Phi$.

Let $Z_{i}, W(i=1, \cdots, n)$ be Banach spaces and let $u$ be an $n$ linear continuous operator from the product of the spaces $Z_{1}, \cdots, Z_{n}$ into the space $W$. Denote the norms in the above spaces by || .

Theorem 2. If $v_{i} \in A^{\Phi}\left(\mu_{i}, Z_{i}\right)$ for $i=1, \cdots, n$ then $v \in A^{\Phi}(\mu, W)$, where

$$
v\left(A_{1} \times \cdots \times A_{n}\right)=u\left(v_{1}\left(A_{1}\right), \cdots, v_{n}\left(A_{n}\right)\right) \text { for } A \in \Sigma .
$$

Proof. We shall establish that $I_{\Phi}(v / k) \leqq 1$ for some positive $k$. Assume throughout that the $\Delta^{\prime}$-constant $M \geqq 1$. Put

$$
w_{j}(\cdot)=\left|v_{j}(\cdot)\right| / N_{\phi}\left(v_{j}\right)
$$

and $b=M^{n}|u| N_{\Phi}\left(v_{1}\right) \cdots N_{\Phi}\left(v_{n}\right)$, and let $\mathscr{F}$ be a finite family of disjoint sets from $\Sigma$. Then we have the following estimates:

$$
\begin{aligned}
& \Sigma\{\Phi(|v(A)| / b \mu(A)) \mu(A): A \in(\mathscr{F})\} \\
\leqq & \Sigma\left\{\Phi\left(\prod_{k=1}^{n} w_{k}\left(A_{k}\right) / M \mu_{k}\left(A_{k}\right)\right) \mu(A): A \in(\mathscr{F})\right\} \\
\leqq & \Sigma\left\{\left[\prod_{k=1}^{n} M \Phi\left(w_{k}\left(A_{k}\right) / M \mu_{k}\left(A_{k}\right)\right)\right] \mu(A): A \in(\mathscr{F})\right\} .
\end{aligned}
$$


Noting that $\Phi(a / M) \leqq \Phi(a) / M$ for $a \geqq 0$ since $\Phi$ is convex and $M \geqq 1$, we conclude that

$$
\begin{aligned}
& \sum\{\Phi(|v(A)| / b \mu(A)) \mu(A): A \in(\mathscr{F})\} \\
\leqq & \prod_{k=1}^{n}\left\{\Sigma \Phi\left(w_{k}(A) / \mu_{k}(A)\right) \mu_{k}(A): A \in \mathscr{F}_{k}^{r}\right\} \\
\leqq & I_{\phi}\left(w_{1}\right) \cdots I_{\phi}\left(w_{n}\right) \leqq 1 .
\end{aligned}
$$

By taking the appropriate supremum, we see that $I_{\Phi}(v / b) \leqq 1$. From this it can easily be seen that $v \in A^{\Phi}(\mu, W)$.

We shall now consider infinite products of vector-valued finitely additive set functions.

Let $T$ be an arbitrary index set, let $\left(\Omega_{t}, \Sigma_{t}, \mu_{t}\right)(t \in T)$ be a family of probability charge spaces and let $(\Omega, \Sigma, \mu)$ be the product space (i.e., $\Omega=X_{T} \Omega_{t}, \Sigma$ is the family of all finite disjoint unions of sets of the form $A=X_{T} A_{t}$ where $A_{t} \in \Sigma_{t}$ and $A_{t} \neq \Omega_{t}$ for at most a finite number of indices $t \in T$, and $\mu(A)=\Pi_{T} \mu_{t}\left(A_{t}\right)$ for all $A \in \Sigma$.) It is clear that the triple $(\Omega, \Sigma, \mu)$ is a probability charge space.

Let $Y, Z_{t}(t \in T), W$ be Banach spaces, let $z_{t}^{\prime} \in Z_{t}$ each be of unit norm, and let $Z^{\prime}=P_{T}\left(Z_{t}, z_{t}^{\prime}\right)$ be the corresponding multiplicative product space. That is, $P_{T}\left(Z_{t}, z_{t}^{\prime}\right)$ is the set of all $z=\left(z_{t}\right)_{T}$ such that $z_{t} \in Z_{t}$ for $t \in T$ and $\Sigma_{T}\left|z_{t}-z_{t}^{\prime}\right|<\infty$. Define a functional $d$ by

$$
d(y, z)=\Sigma_{T}\left|y_{t}-z_{t}\right|
$$

for all $y, z \in Z^{\prime}$. In [3] it is shown that the space $\left(Z^{\prime}, d\right)$ is a complete metric space. It is easy to see that the space $Z^{\prime}$ is also an affine subspace of the linear space $X_{T} Z_{t}$, and if $T$ is uncountable the metric $d$ cannot be extended to a metric on the space $X_{T} Z_{t}$.

An operator $u$ mapping the space $Z^{\prime}$ into the space $W$ shall be called infinitely linear and bounded if $u$ is linear on each coordinate space $Z_{t}$ separately, for all $t \in T$, and if there is a positive constant $c$ such that $|u(z)| \leqq c \Pi_{T}\left|z_{t}\right|$ for all $z \in Z^{\prime}$. Let $\|u\|$ denote the smallest of all such constants. Let $L\left(Z^{\prime} ; W\right)$ denote the space of all such bounded infinitely linear operators. In [3] it is shown that the space $L\left(Z^{\prime} ; W\right)$ under the functional \|\| is a Banach space.

In order to make the next definition we must further restrict the function $\Phi$ so that $\Phi(1)=1$ and the $\Delta^{\prime}$-constant $M=1$. These conditions are the results of applying a version of Lemma 13 of [5], modified to assume the $\Delta^{\prime}$-condition. Since the functions $g$ and $f$ from Remark 1 are nontrivial examples of functions which satisfy these conditions, the additional conditions are not as restrictive as they originally appeared to be.

Select $v_{t}^{\prime} \in A^{\Phi}\left(\mu_{t}, Z_{t}\right)$ such that $v_{t}^{\prime}\left(\Omega_{t}\right)=z_{t}^{\prime}$ and $N_{\varphi}\left(v_{t}^{\prime}\right)=1$, and let 
$P_{T}\left(A^{\Phi}\left(\mu_{t}, Z_{t}\right), v_{t}^{\prime}\right)$ be the corresponding multiplicative product space. We remark that from Lemma 13 of [5] it follows that $v(\Omega) \in Z^{\prime}$ for all $v \in P_{T}\left(A^{\varphi}\left(\mu_{t}, Z_{t}\right), v_{t}^{\prime}\right)$.

For the infinite product ring $\Sigma$ we need some notation similar to the $\mathscr{F}$-product concept for the finite case. With this in mind let $\mathscr{F}$ be a finite family of disjoint sets from the infinite product ring $\Sigma$, let the families $\mathscr{F}_{t}$ and $\mathscr{F}_{t}^{r}$ be defined as in the finite product case, and define the $\mathscr{F}$-product as the corresponding finite family of disjoint sets

$$
X_{T} \mathscr{F}_{t}^{r}=\left\{A=X_{T} A_{t}: A_{t} \in \mathscr{F}_{t}^{r} \text { for all } t \in T\right\}
$$

from the ring $\Sigma$. Again we shall denote the $\mathscr{F}$-product by $(\mathscr{F})$. (It follows from the definition of $\Sigma$ and properties of a ring that $(\mathscr{F})$ consists of a finite number of disjoint sets from $\Sigma$.)

Theorem 3. If $v \in P_{T}\left(A^{\Phi}\left(\mu_{t}, Z_{t}\right), v_{t}^{\prime}\right)$ and $u \in L\left(Z^{\prime} ; W\right)$ then the function $v^{0}$ defined by $v^{\circ}(A)=u(v(A))$ for all $A \in \Sigma$ belongs to the space $A^{\Phi}(\mu, W)$.

Proof. It is clear that the function $v^{0}$ is finitely additive on $\Sigma$, and from the estimate $\left|v^{0}(A)\right| \leqq\|u\| \Pi_{T}\left|v_{t}\left(A_{t}\right)\right|$ for all $A \in \Sigma$ it is clear that $v(A)=0$ when $\mu(A)=0$. To establish that $I_{\phi}(v / k) \leqq 1$ for some constant $k$, put $w_{t}(\cdot)=\left|v_{t}(\cdot)\right| / N_{\Phi}\left(v_{t}\right)$ and $b=\|u\| \Pi_{T} N_{\Phi}\left(v_{t}\right)$ and let $\mathscr{F}$ be a finite family of disjoint sets from $\Sigma$. Applying Lemma 1, which is clearly valid here, we get the following estimates:

$$
\begin{aligned}
& \Sigma\left\{\Phi\left(\left|v^{0}(A)\right| / b \mu(A)\right) \mu(A): A \in(\mathscr{F})\right\} \\
\leqq & \Sigma\left\{\Phi\left(I_{S^{\prime}} w_{t}\left(\Omega_{t}\right) \Pi_{S}\left(w_{t}\left(A_{t}\right) / \mu_{t}\left(A_{t}\right)\right)\right) \mu(A): A \in(\mathscr{F})\right. \\
\leqq & \Sigma\left\{\Pi_{S} \Phi\left(w_{t}\left(A_{t}\right) / \mu_{t}\left(A_{t}\right)\right) \mu(A): A \in(\mathscr{F})\right\} \\
\leqq & \Pi_{S}\left\{\Sigma\left\{\Phi\left(w_{t}(A) / \mu_{t}(A)\right) \mu_{t}(A): A \in \mathscr{F}_{t} r\right\}\right\} \\
\leqq & \Pi_{S} I_{\mathscr{\varphi}}\left(w_{t}\right) \leqq 1 .
\end{aligned}
$$

Hence $v^{0} \in A^{\Phi}(\mu, W)$ and $N_{\Phi}\left(v^{0}\right) \leqq\|u\| \Pi_{T} N_{\Phi}\left(v_{t}\right)$.

REMARK 2. The results in this note remain valid if all rings are relaxed to prerings, with no changes needed in the above proofs to establish this.

REMARK 3. Forthcoming papers will discuss the other formulations of the Fubini theorems for Orlicz spaces in terms of a countably additive nonnegative finite valued set function (called a volume) defined on a prering. The prering, volume combination appears to be the most natural context in which to discuss the Fubini theorems; that it is also a valid context follows from the work of Bogdanowicz, 
which develops an integration theory based upon the prering and volume. See [2] for a suitable bibliography.

\section{REFERENCES}

1. S. Bochner, Additive set functions on groups, Ann. of Math. 40 (1939), 769-799.

2. W. M. Bogdanowicz, Fubini theorems for generalized Lebesgue-Bochner-Stieltjes integral, Proc. Japan Acad. 42 (1966), 979-983.

3. W. M. Bogdanowicz and V. E. Zander, Fubini-Jessen theorems for an infinitelylinear vectorial integral (to appear).

4. M. A. Krasnosel'skii and Ya. B. Rutickii, Convex functions and Orlicz spaces (Translation), Groningen, 1961.

5. J. J. Uhl, Orlicz spaces of finitely additive set functions, Studia Math. 29 (1967), 19-58.

6. - Martingales of vector valued set functions, Pacific J. Math. 30 (1969), $533-548$.

Received February 13, 1970. The research for this paper was supported in part by West Georgia Faculty Research Grant No. 6913.

West Georgia College

Carrollton, Georgia 


\section{PACIFIC JOURNAL OF MATHEMATICS}

\section{EDITORS}

\author{
H. SAMELSON \\ Stanford University \\ Stanford, California 94305 \\ Richard Pierce \\ University of Washington \\ Seattle, Washington 98105
}

\author{
J. DugundJI \\ Department of Mathematics \\ University of Southern California \\ Los Angeles, California 90007 \\ RICHARD ARENS \\ University of California \\ Los Angeles, California 9.0024
}

\section{ASSOCIATE EDITORS}

\begin{tabular}{|c|c|}
\hline E. F. BECKENBACH & K. YoshidA \\
\hline \multicolumn{2}{|c|}{ SUPPORTING INSTITUTIONS } \\
\hline UNIVERSITY OF BRITISH COLUMBIA & STANFORD UNIVERSITY \\
\hline CALIFORNIA INSTITUTE OF TECHNOLOGY & UNIVERSITY OF TOKYO \\
\hline UNIVERSITY OF CALIFORNIA & UNIVERSITY OF UTAH \\
\hline MONTANA STATE UNIVERSITY & WASHINGTON STATE UNIVERSITY \\
\hline UNIVERSITY OF NEVADA & UNIVERSITY OF WASHINGTON \\
\hline NEW MEXICO STATE UNIVERSITY & * \\
\hline OREGON STATE UNIVERSITY & AMERICAN MATHEMATICAL SOCIETY \\
\hline UNIVERSITY OF OREGON & CHEVRON RESEARCH CORPORATION \\
\hline OSAKA UNIVERSITY & TRW SYSTEMS \\
\hline UNIVERSITY OF SOUTHERN CALIFORNIA & NAVAL WEAPONS CENTER \\
\hline
\end{tabular}

The Supporting Institutions listed above contribute to the cost of publication of this Journal, but they are not owners or publishers and have no responsibility for its content or policies.

Mathematical papers intended for publication in the Pacific Journal of Mathematics should be in typed form or offset-reproduced, (not dittoed), double spaced with large margins. Underline Greek letters in red, German in green, and script in blue. The first paragraph or two must be capable of being used separately as a synopsis of the entire paper. The editorial "we" must not be used in the synopsis, and items of the bibliography should not be cited there unless absolutely necessary, in which case they must be identified by author and Journal, rather than by item number. Manuscripts, in duplicate if possible, may be sent to any one of the four editors. Please classify according to the scheme of Math. Rev. Index to Vol. 39. All other communications to the editors should be addressed to the managing editor, Richard Arens, University of California, Los Angeles, California, 90024.

50 reprints are provided free for each article; additional copies may be obtained at cost in multiples of 50 .

The Pacific Journal of Mathematics is published monthly. Effective with Volume 16 the price per volume (3 numbers) is $\$ 8.00$; single issues, $\$ 3.00$. Special price for current issues to individual faculty members of supporting institutions and to individual members of the American Mathematical Society: $\$ 4.00$ per volume; single issues $\$ 1.50$. Back numbers are available.

Subscriptions, orders for back numbers, and changes of address should be sent to Pacific Journal of Mathematics, 103 Highland Boulevard, Berkeley, California, 94708.

PUBLISHED BY PACIFIC JOURNAL OF MATHEMATICS, A NON-PROFIT CORPORATION

Printed at Kokusai Bunken Insatsusha (Internatıonal Academic Printing Co., Ltd.), 7-17, Fujimi 2-chome, Chiyoda-ku, Tokyo, Japan. 


\section{Pacific Journal of Mathematics}

\section{Vol. 35, No. $3 \quad$ November, 1970}

John D. Arrison and Michael Rich, On nearly commutative degree one algebras . . . 533

Bruce Alan Barnes, Algebras with minimal left ideals which are Hilbert spaces . . . . 537

Robert F. Brown, An elementary proof of the uniqueness of the fixed point index . . . 549

Ronn L. Carpenter, Principal ideals in F-algebras .................... 559

Chen Chung Chang and Yiannis (John) Nicolas Moschovakis, The Suslin-Kleene

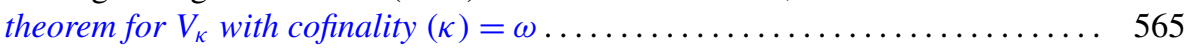

Theodore Seio Chihara, The derived set of the spectrum of a distribution

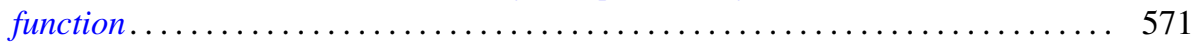

Tae Geun Cho, On the Choquet boundary for a nonclosed subspace of $C(S) \ldots \ldots \quad 575$

Richard Brian Darst, The Lebesgue decomposition, Radon-Nikodym derivative,

conditional expectation, and martingale convergence for lattices of sets .......

David E. Fields, Dimension theory in power series rings . . . . . . . . . . . .

Michael Lawrence Fredman, Congruence formulas obtained by counting

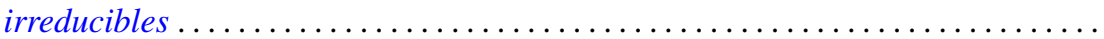

John Eric Gilbert, On the ideal structure of some algebras of analytic functions.....

G. Goss and Giovanni Viglino, Some topological properties weaker than

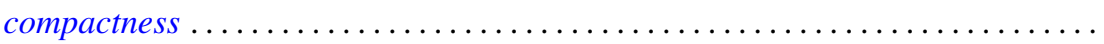

581

601

625

George Grätzer and J. Sichler, On the endomorphism semigroup (and category) of

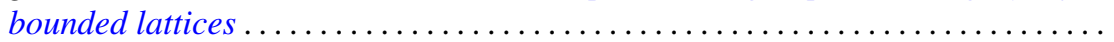

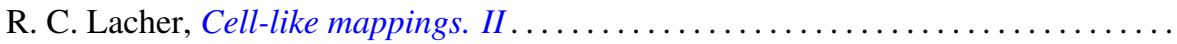

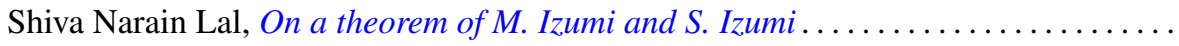

661

Howard Barrow Lambert, Differential mappings on a vector space ...............

Richard G. Levin and Takayuki Tamura, Notes on commutative power joined

semigroups.

Robert Edward Lewand and Kevin Mor McCrimmon, Macdonald's theorem for quadratic Jordan algebras.

J. A. Marti, On some types of completeness in topological vector spaces ....

Walter J. Meyer, Characterization of the Steiner point

717

Saad H. Mohamed, Rings whose homomorphic images are $q$-rings ...

727

Thomas V. O'Brien and William Lawrence Reddy, Each compact orientable surface

of positive genus admits an expansive homeomorphism ...

737

Robert James Plemmons and M. T. West, On the semigroup of binary relations...

743

Calvin R. Putnam, Unbounded inverses of hyponormal operator . .

755

William T. Reid, Some remarks on special disconjugacy criteria for differential

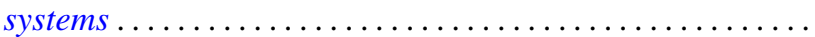

C. Ambrose Rogers, The convex generation of convex Borel sets in euclidean

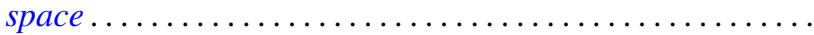

S. Saran, A general theorem for bilinear generating functions .

S. W. Smith, Cone relationships of biorthogonal systems ......

Wolmer Vasconcelos, On commutative endomorphism rings ....

795

Vernon Emil Zander, Products of finitely additive set functions from Orlicz

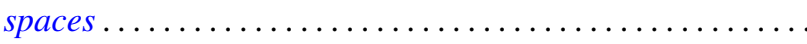

G. Sankaranarayanan and C. Suyambulingom, Correction to: "Some renewal

theorems concerning a sequence of correlated random variables" .

Joseph Zaks, Correction to: "Trivially extending decompositions of $E^{n}$ ”....... 805

Dong Hoon Lee, Correction to: "The adjoint group of Lie groups" ............ 805

James Edward Ward, Correction to: "Two-groups and Jordan algebras". 